\title{
Refractory Evans Syndrome after Autologous Stem Cell Transplantation for Multiple Myeloma: Management with a Second Transplantation
}

\author{
Dai Chihara, Takashi Sakamoto, Kazue Arimoto-Miyamoto, Wataru Kishimoto, \\ Tomoharu Takeoka, Masaaki Tsuji and Tatsuharu Ohno
}

\begin{abstract}
The development of autoimmune disease after autologous stem cell transplantation (ASCT) is very rare in multiple myeloma (MM). We describe the first case of Evans syndrome after ASCT for MM. A 60-year-old man with MM received ASCT and subsequently developed Evans syndrome following two febrile episodes. The syndrome was refractory to conventional therapies but it was managed with a second ASCT. This unique complication was thought to have been triggered by an infection during the recovery of the immune system. We assumed that reconstructing the immune system via ASCT might eliminate infection-induced autoantibodies to platelets and erythrocytes.
\end{abstract}

Key words: multiple myeloma, Evans syndrome, autologous stem cell transplantation

(Inter Med 49: 683-687, 2010)

(DOI: 10.2169/internalmedicine.49.2922)

\section{Introduction}

Recently, autologous stem cell transplantation (ASCT) has become the standard therapy for younger patients with multiple myeloma (1-3). Although several types of autoimmune disorders have been reported after ASCT for various hematologic and non-hematologic disorders (4-10), very few cases of autoimmune disorders related to multiple myeloma $(11,12)$ and only one case complicated with hyperthyroidism after ASCT for multiple myeloma have been reported (7).

Evans syndrome is a rare disease characterized by the simultaneous or sequential development of autoimmune hemolytic anemia (AIHA) and immune thrombocytopenia (ITP) and/or immune neutropenia with no known underlying etiology. The anemia and thrombocytopenia vary with regard to the time of onset, course, and duration. The treatments for Evans syndrome consist of transfusions, corticosteroids, splenectomy, intravenous immunoglobulin, anabolic steroids, vincristine, alkylating agents, cyclosporine, etc (13). Although most cases of Evans syndrome are con- trolled with these treatments, cases refractory to the conventional therapeutic options are occasionally encountered.

Evans syndrome as a complication after high-dose chemotherapy and ASCT is rare. Only three cases of hematological disorders have been reported in the literature $(8,9,14)$, but none have involved multiple myeloma. Naturally, no case has been treated with a second ASCT. Here, we report the first case of refractory Evans syndrome as a complication of ASCT for multiple myeloma that was treated with a second ASCT.

\section{Case Report}

A 60-year-old man visited our hospital with severe back pain and general fatigue in late September 2006. Based on the findings of typical bone lytic changes of the skull, multiple compression fractures of the spine, serum $M$ protein ( $\mathrm{IgG} \lambda)$, renal damage with Bence Jones proteinuria, anemia, and abnormal plasma cells in the bone marrow (plasma

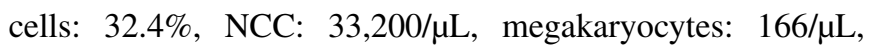
and $\mathrm{M} / \mathrm{E}$ ratio: 1.6), his illness was diagnosed as multiple myeloma. No chromosomal abnormality was observed on 


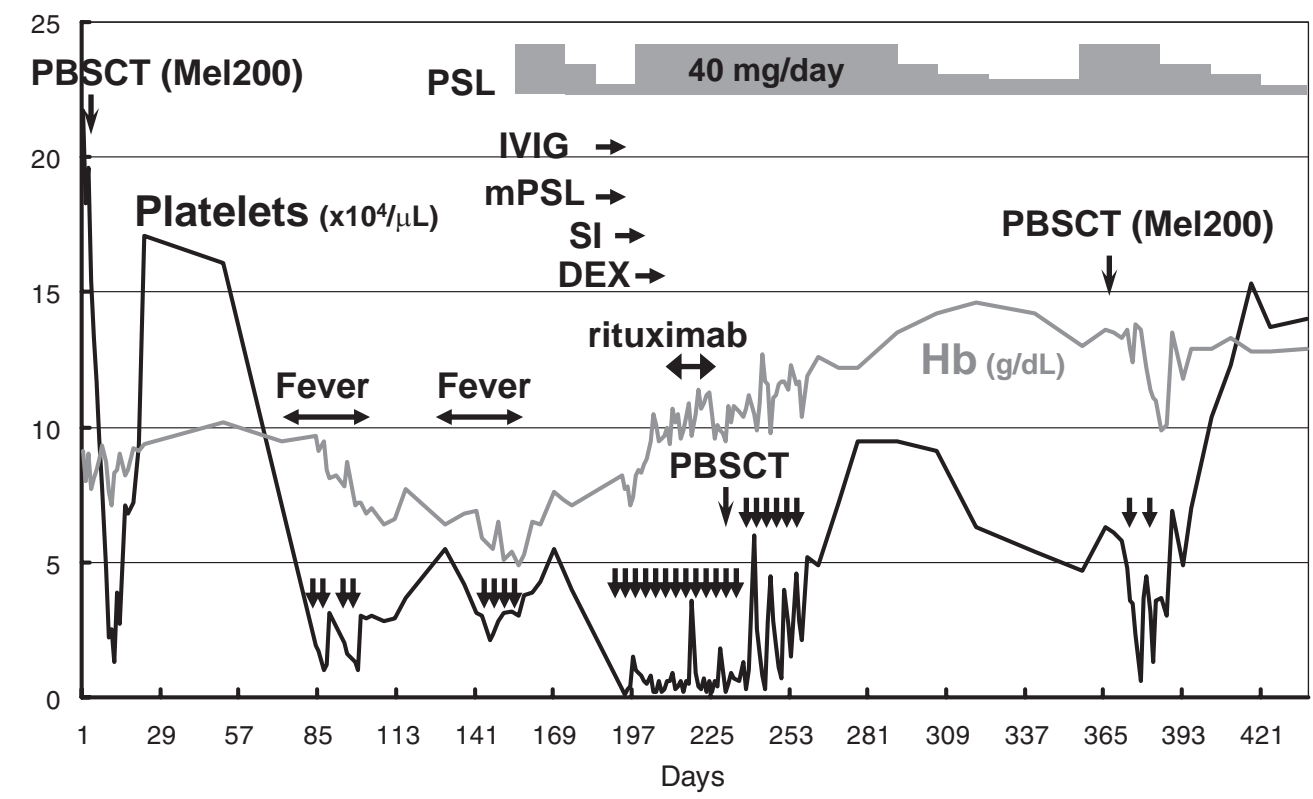

Figure 1. Clinical course of the patient: changes in hemoglobin and platelet levels between the first and second ASCT. The first ASCT was performed on day 3, and the second ASCT was performed on day 367. Mel200: melphalan $100 \mathrm{mg} / \mathrm{m}^{2}$ days -3 and -2, PBSCT: peripheral blood stem cell transplantation, $\downarrow$ : transfusions of PC (A single arrow does not necessarily indicate one transfusion.), IVIG: high-dose intravenous immunoglobulin infusion, PSL: prednisolone, mPSL: methylprednisolone, Dex: dexamethazone, SI: irradiation of the spleen

G-banding. His clinical stage was IIIb according to the Durie-Salmon staging system and II according to the International Staging System. Three courses of VAD (vincristine: $0.4 \mathrm{mg} / \mathrm{m}^{2}$, day $1-4$; doxorubicin: $10 \mathrm{mg} / \mathrm{m}^{2}$, day $1-4$; dexamethasone: $40 \mathrm{mg} / \mathrm{body}$, day 1-4) therapy were effective, and the disease was maintained in a state of very good partial response according to the international uniform response criteria (15). Then, peripheral blood stem cells (PBSC, CD34 ${ }^{+}$cells: $33.7 \times 10^{6} / \mathrm{kg}$ ) were harvested using a mobilization regimen of high-dose cyclophosphamide $\left(2 \mathrm{~g} / \mathrm{m}^{2}\right.$, days 1 and 2). After PBSC collection, he received high dose chemotherapy with melphalan $\left(100 \mathrm{mg} / \mathrm{m}^{2}\right.$, days -3 and -2$)$ followed by autologous stem cell rescue with $6.4 \times 10^{6} / \mathrm{kg}$ $\mathrm{CD}^{+} 4^{+}$cells in mid-January 2007 . His physical and hematological recovery was favorable, and he was discharged from hospital in a state of very good partial response (15).

However, because of two febrile episodes, he was hospitalized in mid-April and in early July ( 3 and 5 months after the first ASCT, respectively). He suffered from a high-grade fever of over $38^{\circ} \mathrm{C}$ that was resistant to antibiotics and lasted for about 3 weeks before disappearing gradually. In both episodes, repeated cultures of blood, urine, stool, and pharyngeal swabs were all negative for specific bacteria, fungi, and parasites. Infection or reactivation of representative viruses such as cytomegalovirus or Epstein-Barr virus was also ruled out. No imaging modality was able to identify the affected area or organ. Although viral infection was assumed to be the likely cause of the febrile episodes because they were refractory to antibiotics and took selflimited courses, the causative agent was not definitely iden- tified.

After the first febrile episode, his anemia gradually progressed with an increase in the number of reticulocytes, and his hemoglobin level reached $4.9 \mathrm{~g} / \mathrm{dL}$ immediately after the second febrile episode (Fig. 1). Bone marrow aspiration revealed erythroid hyperplasia without signs of a relapse of multiple myeloma or megaloblastic changes including giant proerythroblasts (NCC: 125,000/ $\mathrm{LL}, \mathrm{Meg}: 27 / \mu \mathrm{L}$, and M/E ratio: 1.0). Furthermore, elevated serum levels of lactate dehydrogenase (LDH: 358 IU/L) and total bilirubin (T. Bil: $2.54 \mathrm{mg} / \mathrm{dL}$ ) were noted, and his serum haptoglobin level fell below the measurement sensitivity. A direct Coombs test proved positive, and antibodies to parvovirus B19 showed a remote infection pattern. Thus, a diagnosis of AIHA was made. Prednisolone was effective against the AIHA, and immediate improvement of his anemia was observed together with the disappearance of the serum and biochemical abnormalities (Fig. 1).

In late July (six months after the first ASCT and a month after the second febrile episode, when his AIHA was improving owing to steroid therapy), he was admitted again because of severe thrombocytopenia and hemorrhagic diathesis. His platelet count had dropped to $3,000 / \mu \mathrm{L}$, and prominent petechiae of the extremities were noted. A bone marrow examination revealed an increased number of megakaryocytes, without any evidence of dysplastic changes in hematopoietic components or the proliferation of myeloma cells (NCC: 105,000/ $\mathrm{LL}$, Meg: $166 / \mu \mathrm{L}$, and M/E ratio: 2.4), which was consistent with the bone marrow characteristics of ITP. Thus, we diagnosed him with Evans 
syndrome.

Although treatment with high-dose intravenous immunoglobulin (IVIG: $0.4 \mathrm{~g} / \mathrm{kg} /$ day for five days) and methylprednisolone pulse therapy ( $1 \mathrm{~g} /$ day for three days) was initiated, his platelet counts remained extremely low, and his hemorrhagic diathesis was getting worse (Fig. 1). Transfusions of platelet concentrate $(\mathrm{PC})$ produced only transient and minimal responses. On the other hand, the signs of AIHA improved further after these treatments. His serum levels of LDH, bilirubin, and haptoglobin were normalized. Next, we tried rituximab $\left(375 \mathrm{mg} / \mathrm{m}^{2}, 4\right.$ times weekly), irradiation of the spleen ( 2 Gy/day for five times), and dexamethasone pulse therapy (40 mg/day for three days), but the response was not satisfactory; it only resulted in decreasing the frequency of the PC transfusions (Fig. 1).

The serial bone marrow aspirations performed after the diagnosis of Evans syndrome showed a tendency towards a decrease in the number of megakaryocytes. As an alternative method for inducing megakaryopoiesis, we performed a transfusion of the harvested stem cells without chemotherapy eight months after the first ASCT $\left(\mathrm{CD} 34^{+}\right.$cells: $6.4 \times$ $10^{6} / \mathrm{kg}$ ). As a result, his platelet counts were gradually elevated, but he remained dependent on PC and prednisolone. Furthermore, the dose of prednisolone necessary for maintaining his platelet count around $50,000 / \mu \mathrm{L}$ remained very high (40 mg/day) because his body weight was $48 \mathrm{~kg}$. In mid-January 2008 (twelve months after the first ASCT when his platelet count was confirmed to be dependent on steroid therapy), we performed a second ASCT (CD34 ${ }^{+}$cells: $4.5 \times$ $10^{6} / \mathrm{kg}$ ) with a conditioning regimen of high-dose melphalan $\left(100 \mathrm{mg} / \mathrm{m}^{2}\right.$, days -3 and -2$)$ and expected both the suppression of the multiple myeloma and the reconstruction of his immune system. His hematological recovery was smooth, and, finally, his platelet count recovered to an almost normal level (Fig. 1). After the second ASCT, the dose of prednisolone was tapered successfully. Two months after the second ASCT, he was transferred to the outpatient clinic to be treated with $10 \mathrm{mg} /$ day of prednisolone without prophylactic drugs, such as trimethoprim-sulfamethoxazole and itraconazole, as he occasionally complained when treated with such drugs during his hospitalization.

Although he remained well for the following four months, he regrettably developed pneumocystis pneumonia and died of invasive aspergillosis 7 months after the second ASCT. No signs of multiple myeloma relapse or Evans syndrome were recognized.

\section{Discussion}

The development of autoimmune diseases after autologous or allogeneic stem cell transplantation for hematologic malignancies has been reported to occur at a frequency of between $2 \%$ and $5 \%$, with the onset of complications occurring at a median of 8.5 months after transplantation $(6,16)$. Among these cases, autoimmune thrombocytopenia is the most common autoimmune disease after ASCT. Accordingly, the development of autoimmune disease may not be rare following stem cell transplantation. However, despite an extensive search of the English language literature, we found no report of autoimmune disease after ASCT for multiple myeloma, except for a case complicated with hyperthyroidism (3). Autoimmune thrombocytopenia has only rarely been associated with multiple myeloma itself $(11,12)$. Evans syndrome appears to be a more aggressive and a potentially life-threatening condition than either ITP or AIHA alone (13), and thus, its treatment is sometimes difficult.

Since autoimmune diseases have been reported to improve after allogeneic or autologous stem cell transplantation (17-19), ASCT may be a suitable treatment for relapsed or refractory ITP (20-22) and other autoimmune diseases. In ASCT for autoimmune diseases, the regimens chosen are selected specifically for the depletion of lymphocytes, with the aim of eliminating auto-reactive T-cell clones in the patient (16). Drugs like anti-thymocyte globulin and alemtuzumab are thus useful due to their lymphotoxicity.

Most patients with refractory ITP receive ASCT after splenectomy, but we could not perform splenectomy because of the presence of sustained and severe thrombocytopenia with aggravating hemorrhagic diathesis. It was clear that the transfused platelets were being rapidly destroyed because the responses to PC were invariably minimal and transient. As an alternative approach, we performed splenic irradiation, but it was not effective. We supposed that the spleen was not the major site of the destruction of the transfused platelets in this case because functional ablation of the spleen by irradiation did not raise the patient's platelet count. Although high dose prednisolone after sequential immunosuppressive therapies might have been effective in maintaining the platelet count, we ruled out surgery, due to the possible complications induced by long-lasting immunosuppressive therapy and the anticipation of the poor effectiveness of splenectomy. Similarly, we considered that it was not possible to support the patient's platelet count safely over a long period of time with such a high dose of steroid therapy. Furthermore, we supposed that the situation would not change dramatically even if we used a conventional second-line therapy such as azathioprine, cyclophosphamide, cyclosporine A, etc. Therefore, we decided to use a second ASCT instead of a conventional second-line therapy after recognizing the strong dependency of the thrombocytopenia on high-dose steroid therapy.

Huhn, et al. reported that T-cell depleted ASCT for refractory autoimmune thrombocytopenia was tolerable and feasible (response rate: 57\%) (21). As the present patient was thought not to have a predisposition to autoimmune diseases when his stem cells were harvested, we did not remove the $\mathrm{T}$ cells from the harvest. Although cyclophosphamide has frequently been reported as a conditioning regimen in reports on ASCT for ITP (20-24), we adopted melphalan for myeloablation, in consideration of our patient's primary disease (22). As we thought that it was not Evans syndrome but the multiple myeloma that was the biggest risk to his 
life, we chose high-dose melphalan as the treatment as it has been shown to achieve deeper remission, and the deeper remission often equates to a longer survival $(25,26)$.

The present patient was thought to be in a state of very good partial response for multiple myeloma before the second ASCT was performed. The evidence for the efficacy of tandem ASCT vs. single ASCT in patients with multiple myeloma is conflicting. Patients do not always benefit from a second ASCT, because Attal et al concluded that tandem transplantation improves overall survival among patients with myeloma, especially those who do not attain a very good partial response after undergoing single transplantation (25). A recent meta-analysis showed that the use of tandem ASCT did not result in improved overall survival or event-free survival in previously untreated multiple myeloma. However, it was also concluded that tandem ASCT is associated with improved response rates but it is also a risk of a clinically significant increase in therapyrelated mortality (26). Thus, we expected to achieve a more stringent effect on the multiple myeloma as well as beneficial effects on the patient's Evans syndrome with the second ASCT.

It is unlikely that the first bout of ASCT directly affected the emergence of Evans syndrome in this case. Several theories have been proposed to explain how autoimmune diseases emerge after transplantation (7). These include a transient imbalance of the immune system during the posttransplant period and viral infections that occur during the post-transplant period (27). In this patient, the febrile episodes that occurred after the first ASCT were thought to have been caused by a viral infection and might have caused the autoimmunity. The second ASCT was effective at treating Evans syndrome, at least in the short term. We assume that, due to the reconstruction of the immune system with ASCT, infection-induced autoantibodies to platelets and erythrocytes were eliminated. In other words, the second ASCT was successful in resetting the immune system, which had been distorted by an infection in the fragile early phase after the first ASCT. It might be possible that similar autoimmune phenomenon takes place if similar febrile episode interrupts normal recovery of the immune system in the early phase after the second ASCT. Although we could not estimate the long-term efficacy of our treatment against the primary disease in this case, the refractory Evans syndrome appeared to be well controlled by the second ASCT. The patient had received chemotherapy and immunosuppressive therapies for a long period of time and had undergone multiple ASCT since the diagnosis of multiple myeloma was made, and, to make matters worse, he refused prophylactic therapy in the outpatient clinic. His long-lasting immunosuppressive state without prophylaxis may have been a remote cause of his fatal infection. Further studies are needed to explore the efficacy of stem cell transplantations against refractory autoimmune diseases and to clarify the pathogenetic mechanisms of autoimmune diseases associated with stem cell transplantation.

\section{References}

1. Child JA, Morgan GJ, Davies FE, et al. High-dose chemotherapy with hematopoietic stem-cell rescue for multiple myeloma. N Engl J Med 348: 1875-1883, 2003.

2. Attal M, Harousseau JL, Stoppa AM, et al. A prospective, randomized trial of autologous bone marrow transplantation and chemotherapy in multiple myeloma. Intergroupe Francais Du Myelome. N Engl J Med 335: 91-97, 1996.

3. Hahn T, Wingard JR, Anderson KC, et al. The role of cytotoxic therapy with hematopoietic stem cell transplantation in the therapy of multiple myeloma: an evidence-based review. Biol Blood Marrow Transplant 9: 4-37, 2003.

4. Jillella AP, Kallab AM, Kutlar A. Autoimmune thrombocytopenia following autologous hematopoietic cell transplantation: review of literature and treatment options. Bone Marrow Transplant 26: 925 927, 2000.

5. Ahmad I, Haider K, Kanthan R. Autoimmune thrombocytopenia following tandem autologous peripheral blood stem cell transplantation for refractory germ cell tumor. Bone Marrow Transplant 34: 279-280, 2004.

6. Hequet O, Salles G, Ketterer N, et al. Autoimmune thrombocytopenic purpura after autologous stem cell transplantation. Bone Marrow Transplant 32: 89-95, 2003.

7. Ishikawa F, Shigematsu H, Gondo H, Okamura T, Niho Y. Autoreactive antibodies following autologous peripheral blood stem cell transplantation. Bone Marrow Transplant 22: 729-731, 1998.

8. Keung YK, Cobos E, Bolanos-Meade J, Issarachai S, Brideau A, Morgan D. Evans syndrome after autologous bone marrow transplant for recurrent Hodgkin's disease. Bone Marrow Transplant 20: 1099-1101, 1997.
9. Kamezaki K, Fukuda T, Makino S, Harada M. Evans' syndrome following autologous peripheral blood stem cell transplantation for non-Hodgkin's lymphoma. Clin Lab Haematol 26: 291-293, 2004.

10. Lambertenghi-Deliliers GL, Annaloro C, Della Volpe A, Oriani A, Pozzoli E, Soligo D. Multiple autoimmune events after autologous bone marrow transplantation. Bone Marrow Transplant 19: 745747, 1997.

11. Verdirame JD, Feagler JR, Commers JR. Multiple myeloma associated with immune thrombocytopenic purpura. Cancer 56: 1199$1200,1985$.

12. Gupta V, Hegde UM, Parameswaran R, Newland AC. Multiple myeloma and immune thrombocytopenia. Clin Lab Haematol 22: 239-242, 2000.

13. Norton A, Roberts I. Management of Evans syndrome. Br J Haematol 132: 125-137, 2006.

14. Gonzalez-Vicent M, Diaz MA, Garcia-Sanchez F, Molina J, Madero L. Early onset of Evans syndrome following autologous peripheral blood progenitor cell transplantation in a child. Haematologica 87: ECR17, 2002.

15. Durie BG, Harousseau JL, Miguel JS, et al. International uniform response criteria for multiple myeloma. Leukemia 20: 1467-1473, 2006.

16. Loh Y, Oyama Y, Statkute L, et al. Development of a secondary autoimmune disorder after hematopoietic stem cell transplantation for autoimmune diseases: role of conditioning regimen used. Blood 109: 2643-2648, 2007.

17. Yin JA, Jowitt SN. Resolution of immune-mediated diseases following allogeneic bone marrow transplantation for leukaemia. Bone Marrow Transplant 9: 31-33, 1992. 
18. Marmont AM, Van Bekkum DW. Stem cell transplantation for severe autoimmune diseases: new proposals but still unanswered questions. Bone Marrow Transplant 16: 497-498, 1995.

19. Snowden JA, Biggs JC, Brooks PM. Autologous blood stem cell transplantation for autoimmune diseases. Lancet 348: 1112-1113, 1996.

20. Skoda RC, Tichelli A, Tyndall A, Hoffmann T, Gillessen S, Gratwohl A. Autologous peripheral blood stem cell transplantation in a patient with chronic autoimmune thrombocytopenia. Br J Haematol 99: 56-57, 1997.

21. Huhn RD, Fogarty PF, Nakamura R, et al. High-dose cyclophosphamide with autologous lymphocyte-depleted peripheral blood stem cell (PBSC) support for treatment of refractory chronic autoimmune thrombocytopenia. Blood 101: 71-77, 2003.

22. Elli E, Parma M, Perseghin P, et al. Successful treatment with Tdepleted autologous peripheral blood stem cell transplantation of refractory chronic autoimmune thrombocytopenic purpura. Haematologica 92: e7-e8, 2007.
23. Statkute L, Verda L, Oyama Y, et al. Mobilization, harvesting and selection of peripheral blood stem cells in patients with autoimmune diseases undergoing autologous hematopoietic stem cell transplantation. Bone Marrow Transplant 39: 317-329, 2007.

24. Passweg JR, Rabusin M, Musso M, et al. Haematopoetic stem cell transplantation for refractory autoimmune cytopenia. Br J Haematol 125: 749-755, 2004.

25. Attal M, Harousseau JL, Facon T, et al. Single versus double autologous stem-cell transplantation for multiple myeloma. N Engl J Med 349: 2495-2502, 2003.

26. Kumar A, Kharfan-Dabaja MA, Glasmacher A, Djulbegovic B. Tandem versus single autologous hematopoietic cell transplantation for the treatment of multiple myeloma: a systematic review and meta-analysis. J Natl Cancer Inst 101: 100-106, 2009.

27. Garcia Vela JA, Ona F, Monteserin MC, et al. Autoimmune thrombocytopenia after autologous bone marrow transplantation. Am J Hematol 46: 375, 1994.

(C) 2010 The Japanese Society of Internal Medicine

http://www.naika.or.jp/imindex.html 